\title{
Similarities and Differences of Strategies between Bipolar and Depressive Disorders on Stress Coping
}

\author{
Hwagyu Suh', Tae Uk Kang ${ }^{2}$, Eunsoo Moon ${ }^{1,3} \bowtie$, Je Min Park ${ }^{1,3}$, Byung Dae Lee ${ }^{1,3}$, \\ Young Min Lee ${ }^{1,3}$, Hee Jeong Jeong ${ }^{1}$, Soo Yeon Kim¹, Kangyoon Lee', and Hyun Ju Lim¹ \\ ${ }^{1}$ Department of Psychiatry and Medical Research Institute, Pusan National University Hospital, Busan, Republic of Korea \\ ${ }^{2}$ Department of Psychiatry, Seoul Metropolitan Eunpyeung Hospital, Seoul, Republic of Korea \\ ${ }^{3}$ Department of Psychiatry, Pusan National University School of Medicine, Yangsan, Republic of Korea
}

\begin{abstract}
Objective As coping strategies can influence the illness course of mood disorder, they could be potential targets for psychological intervention. The current study investigated the similarities and differences in stress coping styles between bipolar disorder (BD) and depressive disorder (DD).

Methods Subjects with BD $(n=135)$ and DD $(n=100)$ who met the DSM-IV diagnostic criteria were included in this analysis. Coping strategies were assessed using the coping inventory for stressful situations and depressive symptoms were assessed by Beck depression inventory.

Results The BD group showed significantly more avoidant and task-oriented coping than the DD group $(t=2.714, p=0.007 ; t=2.193$, $\mathrm{p}=0.039$ ). After excluding the effect of the depressive symptoms themselves (by comparing two groups in non-depressive state), the BD group still showed significantly more avoidant and task-oriented coping than the DD group $(\mathrm{t}=2.040, \mathrm{p}=0.045 ; \mathrm{t}=2.556, \mathrm{p}=0.013$ ), but when the symptoms of depression get greater, the difference between BD and DD coping strategies were reduced.

Conclusion Subjects with BD tend to use more task and avoidant coping than DD subjects. But when the symptoms of depression get greater, the difference in coping strategies between $\mathrm{BD}$ and $\mathrm{DD}$ were reduced.

Psychiatry Investig 2020;17(1):71-77
\end{abstract}

Key Words Stress, Coping skills, Mood disorder, Depressive disorder, Bipolar disorder.

\section{INTRODUCTION}

Given that a large portion of mood disorders have recurrent courses, it is important to find and control the factors that predict recurrence and relapse of mood episodes. Adverse life events can have negative effects on onset and course of mood disorders, including depressive disorder (DD) and bipolar disorder (BD). ${ }^{1,2}$ For this reason, how to coping with daily stressor can be important in managing mood.

As a part of adaptational processes, people tend to develop habitual methods of managing stressful situations and coping with their emotional turmoil. ${ }^{3}$ In most cases, these habitual

Received: June 20, 2019 Revised: August 13,2019

Accepted: October 7, 2019

$\square$ Correspondence: Eunsoo Moon, MD, $\mathrm{PhD}$

Department of Psychiatry and Medical Research Institute, Pusan National University Hospital, Pusan National University School of Medicine, 179 Gudeok-ro, Seo-gu, Busan 49241, Republic of Korea

Tel: +82-51-240-7303, Fax: +82-51-248-3648, E-mail: esmun@hanmail.net

(a) This is an Open Access article distributed under the terms of the Creative Commons Attribution Non-Commercial License (https://creativecommons.org/licenses/bync/4.0) which permits unrestricted non-commercial use, distribution, and reproduction in any medium, provided the original work is properly cited. methods will help individuals manage stressful situations, but they are not all equally efficient at this task. Some methods only can make matters worse. ${ }^{4}$ In this reason, it may be important to identify these habitual ways of coping, assess how effectively this will handle stressful situations, and correct them if necessary. ${ }^{5}$

Endler and Parker have identified three coping styles: taskoriented, emotion-oriented and avoidance-oriented coping. Task-oriented coping describes task-oriented efforts aimed at solving the problem, cognitively restructuring the problem, or attempts to alter the situation. Emotion-oriented coping describes emotional reactions that are self-oriented, aimed to reduce stress. It includes emotional responses such as selfblaming, getting angry and rumination. Avoidance-oriented coping describes activities and cognitive changes aimed at avoiding stressful situation and alleviate stress. ${ }^{6}$

Research indicated that patients with depressions tend to use maladaptive or emotion-oriented strategies. ${ }^{7.8}$ Problem solving (task-oriented coping) was related to less depression and emotion discharge (emotion-oriented coping) was asso- 
ciated with depression. ${ }^{9}$ Other researchers found that emotion and avoidance-oriented coping are positively correlated with an increase in depressive symptomatology while task-oriented coping is negatively correlated with the later variable. ${ }^{10,11}$

Most of the studies on the coping in mood disorders were conducted with major depressive disorder patients. A recent review of emotion regulation strategies in $\mathrm{BD}$ concluded that $\mathrm{BD}$ endorses putatively maladaptive strategies for regulating negative affect (i.e., rumination, self-blame, suppression and catastrophizing) more than non-clinical controls. ${ }^{12}$ Another study on coping in $\mathrm{BD}$ found that bipolar patients were more likely to ruminate about positive affect and engage in risk taking than DD patients when they are faced with negative affect. Coping style differences were even observed between BD subtypes (Bipolar I and II). ${ }^{13}$

These findings suggest that DD and BD should use different coping strategies which could be affected by mood states and vice versa. To date, however, no studies to our knowledge have examined coping styles, mood states and their inter-relationship in both DD and BD clinical sample. Observing the longitudinal course $\mathrm{BD}$, depressive episodes and symptoms prevail over manic or hypomanic episode. ${ }^{14}$ Therefore, it was considered that the study of depressive episode, which occupies most of the natural history of the disease, would be more useful in the study of coping with stress.

This study aimed to determine the similarities and differences in the coping styles between DD and BD, in association with severity of depression. Moreover, as culture is a fundamental context in shaping coping styles of individual, and since only a few studies on coping with stress in Koreans have been conducted to date, this study on stress coping in Koreans will be more meaningful.

\section{METHODS}

\section{Subjects}

A total of 235 subjects aged between 18 and 60 with bipolar disorder $(n=135)$ and depressive disorder $(n=100)$ were referred among the subjects visited the mood disorder clinic in the Department of Psychiatry at Pusan National University Hospital between January 2012 and January 2018. All were diagnosed at intake by trained psychiatric clinicians, according to the Diagnostic and Statistical Manual of Mental disorder 4th edition (DSM-IV-TR). ${ }^{15}$ Subjects with a past history of a major depressive disorder who meet all criteria for hypomania except the duration criterion, and subjects with sufficient duration but insufficient symptoms of hypomania are present to meet criteria for the bipolar II disorder were categorized as bipolar disorder not otherwise specified (BD-NOS). Depressive disorder not otherwise specified (DD-NOS) was diagnosed when the depressive disorder does not meet the full criteria for major depressive disorder or dysthymia.

Exclusion criteria in this study were schizoaffective disorder, mental retardation, organic mental disorders, subjects having serious medical illness and subjects who were illiterate or visually impaired. As our study used self-reporting scale and due to the difficulty of responding to the self-report scale and the reliability of the responses, we also excluded subjects with severe symptoms (scored more than 6 in the clinical global impression-severity scale).

The bipolar group $(n=135)$ consisted of 49 subjects diagnosed with bipolar I disorder (36.3\%), 30 subjects with bipolar II disorder (22.2\%), and 56 subjects with BD-NOS (41.5\%). The depressive group $(\mathrm{n}=100)$ consisted of 63 major depressive disorder subjects (63.0\%), 7 dysthymia subjects (7.0\%), and 30 DD-NOS subjects (30.0\%) (Table 1).

Their clinical variables were checked by using electronic medical records, and this study was approved by the Pusan National University Hospital Institutional Review Board (H1903-019-077).

\section{Procedures}

We compared the stress-coping styles between bipolar disorder subjects and depressive disorder subjects. Sociodemographic variables such as age, sex, education years, job and marital status were examined. Coping strategies were assessed using the Coping Inventory for Stressful Situations (CISS). Depressive symptoms were assessed by Beck Depression Inventory (BDI). The data used in this study were all collected on the first visit.

\section{Coping inventory for stressful situation (CISS)}

The CISS is useful tool for understanding the important interaction between stressful events and how to handle them. CISS is a self-reporting scale to assess emotion-, task-, and avoidance-oriented stress coping styles developed by Endler and Parker. ${ }^{16}$ Respondents are asked to "indicate how much you engage in these types of activities when you encounter a difficult, stressful, or upsetting situation." Each domain contains 16 items and total of 48 items were quantified by 5-point scales (1='not at all'; 5='very much'). Task domain describes intentional task-oriented efforts or attempt to change the situation for the purpose of solving a problem. Emotion domain refers to a self-oriented emotional reactions to reduces stress. It includes emotional responses (e.g., blame myself for being too emotional, get angry), self-preoccupation, and fantasizing. Avoidance domain describes activities and cognitive changes to avoid stressful situation. In this study, we used the Korean version of CISS adapted by Park et al. ${ }^{17,18}$ 
Table 1. Sociodemographic and clinical characteristics of the subjects

\begin{tabular}{|c|c|c|c|c|}
\hline & Bipolar disorder $(\mathrm{N}=135)$ & Unipolar disorder $(\mathrm{N}=100)$ & t or $\chi^{2}$ & $\mathrm{p}$ \\
\hline \multicolumn{5}{|c|}{ Sociodemographic characteristics } \\
\hline Mean age, year & $31.4(11.5)$ & $34.7(13.9)$ & -1.961 & 0.051 \\
\hline Education, year & $13.8(2.0)$ & $13.6(2.4)$ & 0.497 & 0.619 \\
\hline Sex, & & & 3.065 & 0.080 \\
\hline Male & $56(41.5)$ & $53(53.0)$ & & \\
\hline Female & $79(58.5)$ & $47(47.0)$ & & \\
\hline Job & & & 1.051 & 0.591 \\
\hline No & $33(24.4)$ & $26(26.0)$ & & \\
\hline Part time & $72(53.3)$ & $47(47.0)$ & & \\
\hline Full time & $30(22.2)$ & $27(27.0)$ & & \\
\hline Marital status & & & 0.975 & 0.614 \\
\hline Unmarried & $86(63.7)$ & $59(59.0)$ & & \\
\hline Married/cohabited & $34(25.2)$ & $31(31.0)$ & & \\
\hline Divorced/separated & $15(11.1)$ & $10(10.0)$ & & \\
\hline \multicolumn{5}{|l|}{ Clinical characteristics } \\
\hline \multicolumn{5}{|l|}{ Diagnosis } \\
\hline BD-I & $49(36.3)$ & - & - & - \\
\hline BD-II & $30(22.2)$ & - & & \\
\hline BD-NOS & $56(41.5)$ & - & & \\
\hline MDD & - & $63(63.0)$ & & \\
\hline Dysthymia & - & $7(7.0)$ & & \\
\hline DD-NOS & - & $30(30.0)$ & & \\
\hline \multicolumn{5}{|l|}{ CISS score } \\
\hline Task & $40.4(13.9)$ & $37.0(10.0)$ & 2.193 & 0.029 \\
\hline Emotion & $44.1(12.7)$ & $44.7(11.6)$ & -0.360 & 0.719 \\
\hline Avoidance & $40.1(11.1)$ & $36.4(9.4)$ & 2.714 & 0.007 \\
\hline BDI score (cut-off: 18) & $23.1(14.6)$ & $27.6(12.7)$ & -2.465 & 0.014 \\
\hline Non-depressive & $57(42.2)$ & $24(24.0)$ & & \\
\hline Depressive & $78(57.8)$ & $76(76.0)$ & & \\
\hline
\end{tabular}

Mean (SD) or $\mathrm{n}(\%)$. ns: not significant; independent t test or $\chi^{2}$ test. CISS: Coping inventory for stressful situations, BDI: Beck depression inventory, BD-1: bipolar I disorder, BD-II: bipolar II disorder, BD NOS: bipolar disorder not otherwise specified, MDD: major depressive disorder, DD NOS: depressive disorder not otherwise specified

\section{Beck depression inventory (BDI)}

BDI is a widely used 21-item self-report inventory measuring the severity of depression. ${ }^{19}$ Each answer is scored on a scale value of 0 to 3 . Scores lower than 13 refers to minimal symptoms, 14 to 19 mild symptoms, 20 to 28 moderate symptoms and over 29 refer to severe depressive symptoms. According to the validation study of BDI in Korean version, a cut-off score of 18 was recommended as the criterion for the presence of depression. ${ }^{20}$ We used this cut-off value to divide subgroup by their mood states for each group (BD_non-depressive; BD_ depressive; DD_non-depressive; DD_depressive).

\section{Statistics}

In order to compare the variables between bipolar disorder subjects and depressive disorder subjects, chi-square test was used for categorical variables, and independent $t$-test was used for continuous variables. Associations between CISS and sociodemographic, clinical variables were assessed by Pearson correlation. We used analysis of covariance (ANCOVA) with associated sociodemographic and clinical covariates to compare stress-copying styles between bipolar disorder subjects and depressive disorder subjects. The Statistical Package for the Social Science (SPSS) was used to perform the statistical analyses (version 22.0 for Windows, IBM Corp., Armonk, NY, 
USA). In all analyses, the level of significance was met at $\mathrm{p}<$ 0.05, 2-tailed probability.

\section{RESULTS}

\section{Sociodemographic and clinical characteristics of the sample}

Sociodemographic and clinical characteristics of participants in this study are shown in Table 1.

There was a significant difference in BDI score $(\mathrm{p}=0.014)$. Mean BDI score of bipolar disorder group was 23.1 ( \pm 14.6$)$, and $27.6( \pm 12.7)$ in depressive disorder group. However, there was no significant difference in sociodemographic variables between two groups.

Of the 234 subjects recruited, 79 subjects (58.5\%) with BD were female, and 47 subjects (47.0\%) with DD were female. The mean age of the BD group was $31.4( \pm 11.5)$ years and the DD group was $34.7( \pm 13.9)$ years, and the mean education level was $13.8( \pm 2.0)$ years in the BD group and also $13.6( \pm 2.4)$ years in the DD group.

\section{The stress coping styles in subjects with a mood disorder}

Relationships among coping styles and depressive symptomatology in subjects with BD and DD

As we will see later, the symptom severity of depression can affect coping strategies. To separate the effect of the depressive symptoms, each group was divided into depressive (the depressive BD group and the depressive DD group) and non-depressive subgroup (the non-depressive $\mathrm{BD}$ group and the nondepressive DD group).

Coping strategies of BD subjects changed significantly after depression. Depressed BD subjects were less likely to use task and avoidance-oriented coping $(\mathrm{t}=5.572, \mathrm{p}<0.001 ; \mathrm{t}=3.220$, $\mathrm{p}=0.002)$, but more emotion-based coping strategies $(\mathrm{t}=-6.869$, $\mathrm{p}<0.001)$ than the non-depressive BD subjects.

Coping styles of DD subjects were also influenced by their mood. They seemed to use more emotional coping after depression $(\mathrm{t}=-4.932, \mathrm{p}<0.001)$, but other coping strategies were not affected.

\section{Comparisons of stress-coping strategies between the $\mathrm{BD}$ and DD groups}

The BD group showed more avoidant and task-oriented coping than DD group $(\mathrm{t}=2.714, \mathrm{p}=0.007 ; \mathrm{t}=2.193$, $\mathrm{p}=0.039)$. There was no significant difference in emotion-oriented coping (Figure 1A).

As we focused on the difference of the coping strategies between the $\mathrm{BD}$ and $\mathrm{DD}$ groups, we used analysis of covariance (ANCOVA), using the BDI score as a covariate. After adjusting the BDI score, the BD group still showed frequent use of avoidant coping than the DD group ( $\mathrm{p}=0.029)$ (Figure 1B). And when we put age as covariate together, it still showed the same tendency ( $\mathrm{p}=0.062$ ) (Figure 1C). But there was no significant difference in the task-oriented coping $(\mathrm{p}=0.249)$ (Figure $1 \mathrm{~B}$ and $\mathrm{C})$.

\section{Comparison of stress coping strategy between $\mathrm{BD}$ and DD group in non-depressive state}

Previously, we found that BD subjects tend to use more avoidant and task-oriented coping than DD subjects $(\mathrm{t}=2.714, \mathrm{p}=$ $0.007 ; \mathrm{t}=2.193, \mathrm{p}=0.039$ ) (Figure 1A). After excluding the effect of the depressive symptoms themselves (non-depressive state), the $\mathrm{BD}$ group still showed more avoidant and task-oriented coping than the DD group $(\mathrm{t}=2.040, \mathrm{p}=0.045 ; \mathrm{t}=2.556, \mathrm{p}=0.013$ ) (Figure 2A). There was no significant difference in emotion-oriented coping (Figure 2A).
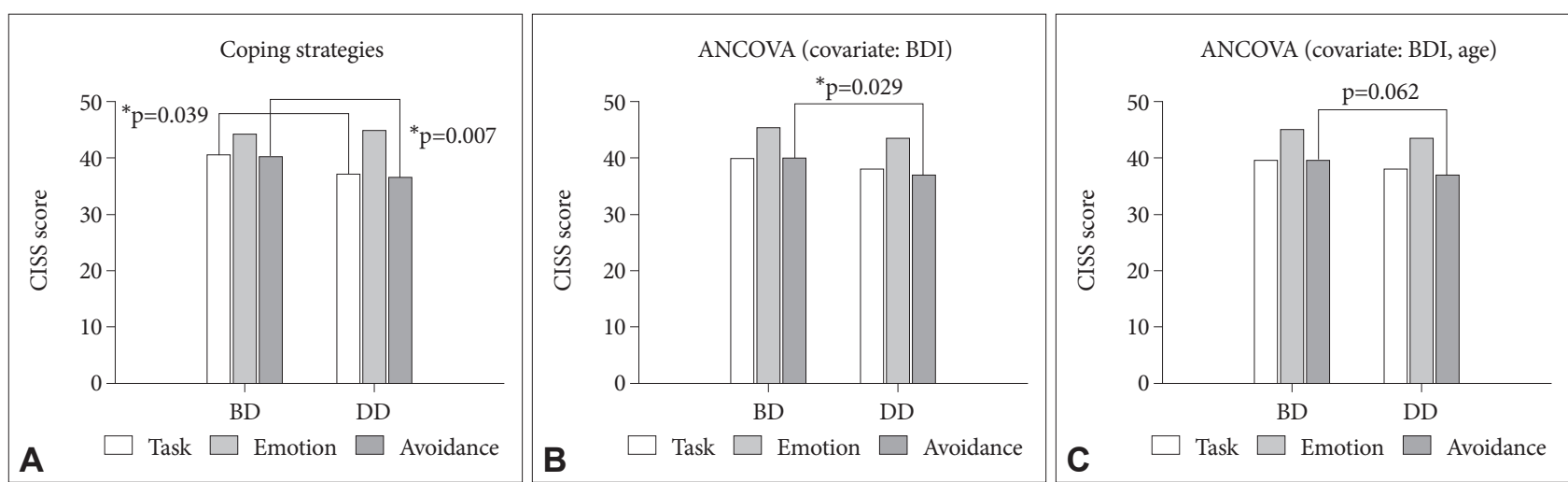

Figure 1. Comparison of the CISS scores between the BD patients and DD patients. BD group showed significantly more avoidant and taskoriented coping than DD group ( $t=2.714, p=0.007 ; t=2.193, p=0.039)(A)$. After adjusting the Beck Depression Inventory (BDI) score by analysis of covariance (ANCOVA), BD group still showed frequent use of avoidant coping than DD group $(p=0.029)(B)$. When age was used as a covariate together, it still showed the same tendency $(p=0.062)(C)$. CISS: Coping Inventory for Stressful Situation, BD: bipolar disorder, DD: depressive disorder. 

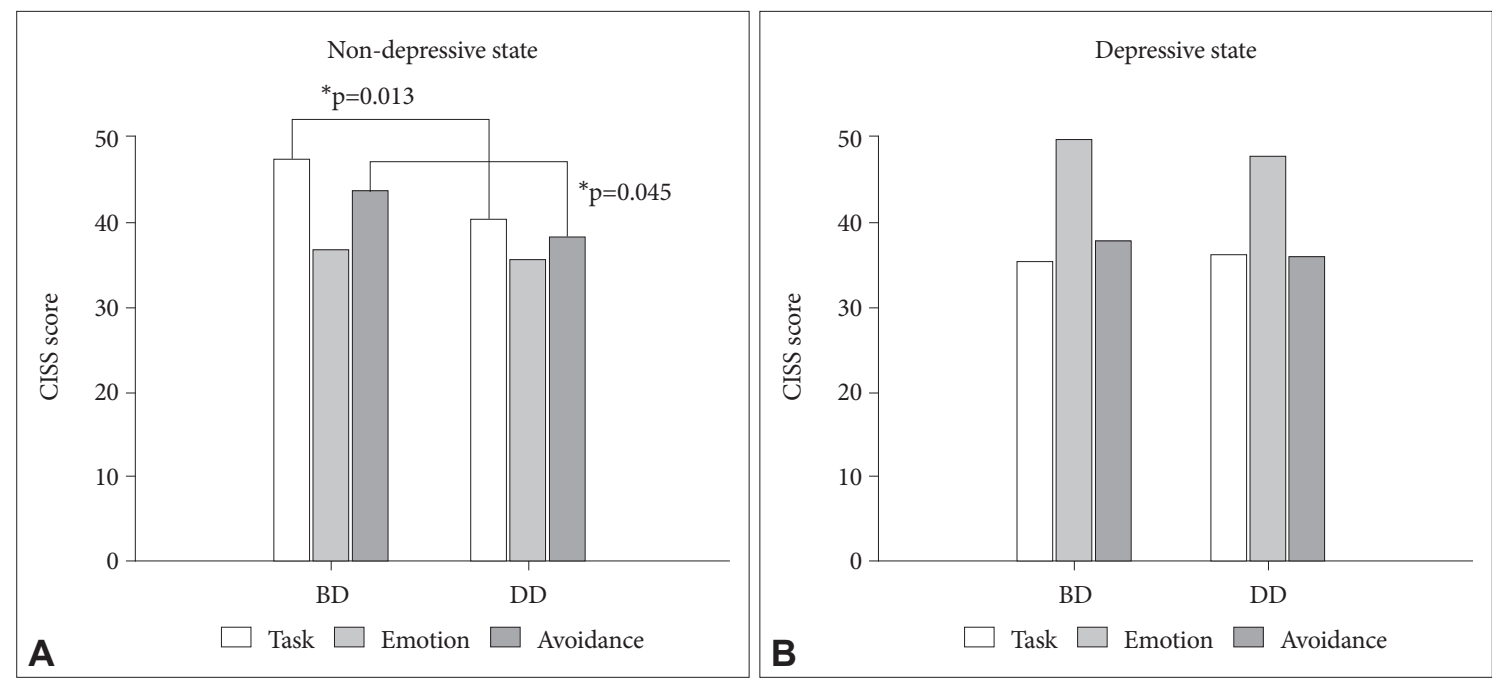

Figure 2. CISS scores between the BD patients and DD patients in non-depressive state and depressive state. The non-depressive BD group showed more avoidant and task-oriented coping than the nondepressive DD group $(t=2.040, p=0.045 ; t=2.556, p=0.013)(A)$. Patients with moderate to severe depressive symptoms (cutoff 18) were separated as depressive group. There was no significant difference in all items of stress coping between Depressive BD group and Depressive DD group (B). CISS: Coping Inventory for Stressful Situation, BD: bipolar disorder, DD: depressive disorder.

\section{Comparison of stress coping strategy between $\mathrm{BD}$ and DD group in depressive state}

Subjects with moderate to severe depressive symptoms (cutoff score of 18 in the Korean version of BDI) were separated as depressive group. There was no significant difference in all items of stress coping between the Depressive $\mathrm{BD}$ group and the Depressive DD group (Figure 2B).

\section{Correlation between the coping styles and the clinical variables in subjects with mood disorder}

To find the relationships between coping styles and depressive symptomatology (BDI score), Pearson correlations were conducted (Table 2). The results revealed significant negative correlations between the severity of depression and both task and avoidance oriented coping styles $(r=-0.429, \mathrm{p}<0.001 ; \mathrm{r}=$ $-0.237, \mathrm{p}<0.001$ ), and a positive correlation between the former variable and emotion oriented coping style $(\mathrm{r}=0.591, \mathrm{p}<0.001)$.

When the correlations were checked in each diagnostic group, task and emotion-oriented coping showed the same correlation patterns. In both $\mathrm{BD}$ and DD group, task-oriented coping was negatively correlated with BDI score $(r=-0.472$, $\mathrm{p}<0.001 ; \mathrm{r}=-0.303, \mathrm{p}=0.002)$ and emotion-oriented coping was positively correlated $(\mathrm{r}=0.596, \mathrm{p}<0.001 ; \mathrm{r}=0.593, \mathrm{p}<0.001)$ while avoidance-related coping showed a significant negative correlation with $\mathrm{BDI}$, only in the $\mathrm{BD}$ group $(\mathrm{r}=-0.270, \mathrm{p}=0.002$; $\mathrm{r}=-0.116, \mathrm{p}=0.251$ ).

We also conducted Pearson correlations with some of the variables (age, education level) that are likely to be relevant to coping. Only the education level showed positive correlations with task-oriented coping $(\mathrm{r}=0.184, \mathrm{p}=0.005)$.
Table 2. Pearson correlation among variables

\begin{tabular}{llll}
\hline & \multicolumn{3}{c}{ CISS score } \\
\cline { 2 - 4 } & \multicolumn{1}{c}{ Task } & \multicolumn{1}{c}{ Emotion } & Avoidance \\
\hline Age & $-0.003(0.962)$ & $-0.118(0.071)$ & $-0.121(0.065)$ \\
Education years & $0.184(0.005)$ & $0.086(0.189)$ & $0.100(0.126)$ \\
BDI score & $-0.429(<0.001)$ & $0.591(<0.001)$ & $-0.237(<0.001)$ \\
BDI (BD) & $-0.472(<0.001)$ & $0.596(<0.001)$ & $-0.270(0.002)$ \\
BDI (DD) & $-0.303(0.002)$ & $0.593(<0.001)$ & $-0.116(0.251)$ \\
\hline
\end{tabular}

Pearson coefficient ( $\mathrm{p}$ value). Pearson correlation analysis. BDI: Beck depression inventory, BD: bipolar disorder, DD: depressive disorder

\section{DISCUSSION}

This study investigated the difference of coping strategies between bipolar disorder patients and depressive disorder patients. To investigate them more precisely, we analyzed the relationship between depression and coping in subjects with mood disorders.

According to the previous studies on the relationship between coping style and depression, increase in depressive symptomatology is associated with fewer task oriented coping behaviors and greater emotion and avoidance oriented coping behaviors. ${ }^{10,11}$ In accordance with the previous studies, our results found a significant negative correlation between the level of depressive symptomatology and task oriented coping styles and a significant positive correlation between the former variable and emotion oriented coping style. On the other hand, in the case of avoidance oriented coping styles, results were opposite to what was expected. The level of depressive 
symptomatology and avoidance oriented coping styles were negatively correlated. These findings were in an agreement with a previous research which conducted with a sample of psychiatric outpatients including all patients regardless of their diagnosis, not only the depressive disorder patients. ${ }^{21}$

Most studies examining the relationship between depressive symptoms and coping styles, subjects were limited to depressive disorder patients, excluding bipolar disorders and other psychiatric disorders. However, in the previous study we mentioned above, subjects were not limited by their diagnosis. Such differences may explain discrepancies between two studies. According to our hypothesis, coping strategies may be different depending on their diagnosis, and the association of coping strategies with depression could be revealed more accurately when we consider their diagnosis.

It was reported that patients with high levels of depressive symptoms use more emotion-oriented coping strategies. ${ }^{10,22}$ Emotion-oriented coping describes emotional reactions that are to reduce stress, yet these coping styles seem to be relatively ineffective in managing the stress and are associated with an increased risk of relapse of mood episodes. ${ }^{23}$ These findings are in line with our results. We found that subjects who are high on depressive symptoms, as assessed by the BDI, engaged in more emotion-oriented coping than those who are low on depressive symptoms in both diagnostic groups (BD and DD).

As we investigated the difference of coping strategies between BD and DD, we found that a more avoidant and taskoriented coping style were used in BD subjects. Especially greater use of avoidant coping was found even after controlling for the severity of depression.

Unlike emotional coping strategies, items of task (e.g., planning, attempts to solve the problem) and some items of avoidance (e.g. seeking social contact) require a high level of energy or activity, which is generally classified as active coping. ${ }^{24}$ In other words, the depressive states mainly using task and avoidance-oriented coping might reflect depression with high level of energy, and it might be associated with a depressive mixed state. The study of Sato et al. ${ }^{25}$ pointed out that depressive mixed state is particularly frequent in depression of BD than depression of DD. Our results might be understood in the same context. However, in order to draw these conclusions, more precise future studies are needed.

Another possible explanation for these findings may relate to their extroversive personality. BD patients tend to have higher levels of extraversion and better social coping than DD patients, ${ }^{26}$ and these characteristics may lead them to use more task and some kind of avoidant coping. ${ }^{24}$

In a study of older age bipolar disorder subjects, better executive functioning was associated to more active coping. ${ }^{27}$ In this study, the Utrecht Coping List (UCL) was used to ac- cess the coping strategies. Before applying these results to our study, we need to compare the items included in each category of the two different scales (the UCL and the CISS). In the study we mentioned above, the subscales of active approach (i.e., finding out all about the problems, considering different solutions to the problems) was interpreted as active coping, and the subscales avoidance (i.e., being totally pre-occupied with the problems, worrying about the past) was interpreted as passive coping. However, the items of the UCL's active approach are classified as task-oriented coping, and items of the UCL's subscales avoidance are classified as emotion-oriented coping in the CISS. Other subscales including problem sharing, palliative reactions of the UCL are categorized as avoidance-oriented coping in the CISS. Therefore, when interpreting the results of the study with the CISS categories, better executive functioning was more associated to task-oriented coping.

Our data has shown a positive correlation between education level and task-oriented coping, and it might be due to a better executive function of the subjects with high education level. A recent study investigated the differences in executive function between drug-naive subjects with depressive episode in both $\mathrm{BD}$ and $\mathrm{DD}$ found that the executive function in $\mathrm{DD}$ patients was worse than in $\mathrm{BD}$ patients. ${ }^{28}$ With the results of those studies, it can be deduced that BD patients have better executive function and use more active coping like task and social diversion of avoidance-oriented coping.

Another interesting new finding is that once the symptoms of depression get greater, the difference between BD and DD coping strategies is reduced. This may suggest that when the depressive symptoms are mild, there is a large difference between the two diagnostic groups when choosing a coping strategy, but when the symptoms gets severe, the subjects of both groups failed to cope with the stress properly and the difference is reduced.

As shown in Figure 2B, in the depressive state, both BD and DD groups used emotion-oriented coping, which is known to be maladaptive, significantly more than task and avoidance. The results of this study do not tell what is the cause or effect, but again confirm that emotion-oriented coping is associated with depression.

This study was limited due to the use of a cross-sectional design, disallowing examination of changes in coping styles over time. The cross-sectional design provided a limited insight into the complex relationships between coping strategies, depressive symptoms and the two diagnostic groups. There is no information on how coping changes when a depressed state changes in each subject, and there is a limit to the interpretation because it cannot be compared with the characteristics of the general population. Second, another limitation is the lack of data on variables that may affect coping, such as cognitive 
patterns, temperament, medication, drug compliance and the time of the first onset. Therefore, the effects of these variables could not be ruled out. A further limitation was the use of selfreport questionnaires, which is inherently more subject to error than clinician-rated measures.

Despite these limitations, the present investigation increases understanding of a relationship between psychiatric diagnosis (BD and $\mathrm{DD}$ ) and their coping strategy. Coping strategy could be a target for selective prevention targeting subgroups. Subgroups can be classified by their age, gender and personality, ${ }^{23}$ and based on the present study, depressive symptom severity and psychiatric diagnosis as well. Recognize the maladaptive coping and changing to the adaptive coping could ameliorate their mood symptoms and change the course of the disease.

Studies about the coping strategies measured coping using various scales, and each of those scales classified coping strategies in different way. This makes interpretation difficult. Developing reliable and valid tools that assesses how people cope with stressful situations and unifying the measurements are necessary. Given the limitations of the present study, longitudinal studies using precise scales for coping styles in $\mathrm{BD}, \mathrm{DD}$ patients and healthy controls are required for further validation.

\section{Acknowledgments}

The results of this study were partly presented at the 19th Annual Conference of the International Society of Bipolar Disorders, May 4-7, 2017, Washington DC, USA.

\section{Conflicts of Interest}

The authors have no potential conflicts of interest to disclose.

\section{Author Contributions}

Conceptualization: Hwagyu Suh, Tae Uk Kang, Eunsoo Moon. Data curation: Hwagyu Suh, Tae Uk Kang, Eunsoo Moon, Hee Jeong Jeong, Soo Yeon Kim, Kangyoon Lee, Hyun Ju Lim. Formal analysis: Hwagyu Suh, Hyun Ju Lim. Investigation: Hwagyu Suh, Tae Uk Kang, Eunsoo Moon. Methodology: Eunsoo Moon, Je Min Park, Byung Dae Lee, Young Min Lee. Project administration: Eunsoo Moon. Resources: Eunsoo Moon. Software: Hwagyu Suh, Hyun Ju Lim. Supervision: Eunsoo Moon, Je Min Park, Byung Dae Lee, Young Min Lee. Validation: Je Min Park, Byung Dae Lee, Young Min Lee. Visualization: Hwagyu Suh. Writing—original draft: Hwagyu Suh, Tae Uk Kang, Eunsoo Moon. Writing_review \& editing: Je Min Park, Byung Dae Lee, Young Min Lee, Hee Jeong Jeong, Soo Yeon Kim, Kangyoon Lee.

\section{ORCID iDs}

$\begin{array}{ll}\text { Eunsoo Moon } & \text { https://orcid.org/0000-0002-8863-3413 } \\ \text { Hwagyu Suh } & \text { https://orcid.org/0000-0002-0360-6708 }\end{array}$

\section{REFERENCES}

1. Ellicott A, Harnincn C, Gitlin M, Brown G, Jamison K. Life events and the course of bipolar disorder. Am J Psychiatry 1990;147:1194-1198.

2. Hunt N, Bruce-Jones W, Silverstone T. Life events and relapse in bipolar affective disorder. J Affect Disord 1992;25:13-20.

3. Lazarus RS. Psychological stress and coping in adaptation and illness. Int J Psychiatry Med 1974;5:321-333.

4. Compas BE, Connor-Smith JK, Saltzman H, Thomsen AH, Wadsworth
ME. Coping with stress during childhood and adolescence: problems, progress, and potential in theory and research. Psychol Bull 2001;127:87.

5. Seiffge-Krenke I. Adaptive and maladaptive coping styles: does intervention change anything? Eur J Develop Psychol 2004;1:367-382.

6. Endler NS, Parker J. Coping Inventory for Stressful Situations. Toronto: Multi-Health Systems Incorporated; 1990.

7. Bruder-Mattson SF, Hovanitz CA. Coping and attributional styles as predictors of depression. J Clin Psychol 1990;46:557-565.

8. Tomczak-Witych A. Coping with stress strategies among female patients suffering from a depression. Psychiatr Polska 2006;40:491-502.

9. Swindle RW, Cronkite RC, Moos RH. Life stressors, social resources, coping, and the 4-year course of unipolar depression. J Abnorm Psychol 1989;98:468-477.

10. Endler NS, Parker JD. State and trait anxiety, depression and coping styles. Aust J Psychol 1990;42:207-220.

11. Billings AG, Moos RH. Psychosocial processes of remission in unipolar depression: Comparing depressed patients with matched community controls. J Consult Clin Psychol 1985;53:314-325.

12. Dodd A, Lockwood E, Mansell W, Palmier-Claus J. Emotion regulation strategies in bipolar disorder: A systematic and critical review. J Affect Disord 2019;246:262-284.

13. Fletcher K, Parker GB, Manicavasagar V. Coping profiles in bipolar disorder. Compr Psychiatry 2013;54:1177-1184.

14. Judd LL, Akiskal HS. Depressive episodes and symptoms dominate the longitudinal course of bipolar disorder. Curr Psychiatry Rep 2003;5:417418.

15. American Psychiatric Association. Diagnostic and Statistical Manual of Mental Disorders-IV-TR. Washington DC: American Psychiatric Pub; 2000.

16. Endler NS, Parker JD. Assessment of multidimensional coping: task, emotion, and avoidance strategies. Psychol Assess 1994;6:50-60.

17. Choi Y, Moon E, Park JM, Lee BD, Lee YM, Jeong HJ, et al. Psychometric properties of the coping inventory for stressful situations in Korean adults. Psychiatry Investig 2017;14:427-433.

18. Park Y, Kim K, Noh S. Validity assessment of the CISS (Coping Inventory for Stressful Situation) in Korean high school students. J Korean Neuropsychiatr Assoc 2000;39:55-64.

19. Beck AT, Ward CH, Mendelson M, Mock J, Erbaugh J. An inventory for measuring depression. Arch Gen Psychiatry 1961;4:561-571.

20. Lim SY, Lee EJ, Jeong SW, Kim HC, Jeong CH, Jeon TY, et al. The validation study of Beck Depression Scale 2 in Korean version. Anxiety Mood 2011;7:48-53.

21. Turner RA, King PR, Tremblay PF. Coping styles and depression among psychiatric outpatients. Pers Individ Diff 1992;13:1145-1147.

22. Orzechowska A, Zajączkowska M, Talarowska M, Gałecki P. Depression and ways of coping with stress: a preliminary study. Med Sci Monit 2013;19:1050-1056.

23. Christensen MV, Kessing LV. Clinical use of coping in affective disorder, a critical review of the literature. Clin Pract Epidemiol Ment Health 2005; 1:20.

24. Campbell-Sills L, Cohan SL, Stein MB. Relationship of resilience to personality, coping, and psychiatric symptoms in young adults. Behav Res Ther 2006;44:585-599.

25. Sato T, Bottlender R, Schröter A, Möller HJ. Frequency of manic symptoms during a depressive episode and unipolar 'depressive mixed stateas bipolar spectrum. Acta Psychiatr Scand 2003;107:268-274.

26. Coulston CM, Bargh DM, Tanious M, Cashman EL, Tufrey K, Curran $\mathrm{G}$, et al. Is coping well a matter of personality? A study of euthymic unipolar and bipolar patients. J Affect Disord 2013;145:54-61.

27. Paans NP, Dols A, Comijs HC, Stek ML, Schouws SN. Associations between cognitive functioning, mood symptoms and coping styles in older age bipolar disorder. J Affect Disord 2018;235:357-361.

28. Yang T, Zhao GQ, Mao RZ, Huang J, Xu XR, Su YS, et al. The association of duration and severity of disease with executive function: differences between drug-naïve patients with bipolar and unipolar depression. J Affect Disord 2018;238:412-417. 